\title{
An Analysis Technique/Automated Tool for Comparing and Tracking Analysis Modes of Different Finite Element Models
}

\author{
Robert L. Towner ${ }^{1}$ and Jonathan L. Band ${ }^{2}$ \\ Jacobs Engineering, ESTS Group, Huntsville. AL, 35806
}

\begin{abstract}
An analysis technique was developed to compare and track mode shapes for different Finite Element Models. The technique may be applied to a variety of structural dynamics analyses, including model reduction validation (comparing unreduced and reduced models), mode tracking for various parametric analyses (e.g., launch vehicle model dispersion analysis to identify sensitivities to modal gain for Guidance, Navigation, and Control), comparing models of different mesh fidelity (e.g., a coarse model for a preliminary analysis compared to a higher-fidelity model for a detailed analysis) and mode tracking for a structure with properties that change over time (e.g., a launch vehicle from liftoff through end-of-burn, with propellant being expended during the flight). Mode shapes for different models are compared and tracked using several numerical indicators, including traditional Cross-Orthogonality and Modal Assurance Criteria approaches, as well as numerical indicators obtained by comparing modal strain energy and kinetic energy distributions. This analysis technique has been used to reliably identify correlated mode shapes for complex Finite Element Models that would otherwise be difficult to compare using traditional techniques. This improved approach also utilizes an adaptive mode tracking algorithm that allows for automated tracking when working with complex models and/or comparing a large group of models.
\end{abstract}

\section{Nomenclature}

$\begin{array}{ll}\text { ESE } & =\text { Element Strain Energy } \\ \text { ESTS } & =\text { Engineering, Science, and Technical Services } \\ \text { FEM } & =\text { Finite Element Model } \\ G N \& C & =\text { Guidance, Navigation and Control } \\ G P K E & =\text { Grid Point Kinetic Energy } \\ M A C & =\text { Modal Assurance Criteria } \\ M S F C & =\text { Marshall Space Flight Center } \\ \text { RSS } & =\text { Root Sum Squared } \\ X O R & =\text { Cross-Orthogonality }\end{array}$

\section{Introduction}

$\mathrm{V}$ arious structural dynamics and loads analyses require a detailed comparison of the natural frequencies and mode shapes for different Finite Element Models (FEMs) in order to identify the corresponding modes. The analysis FEMs being compared may differ in a variety of ways, including a revised mesh (as for a higher-fidelity model being compared to a coarser model), a model reduction (as for a model reduced via Guyan Reduction compared to the unreduced model), a model with a consistent mesh that has mass that changes over time (as for a launch vehicle that expends propellant during flight compared to the initial lift-off model), or a model that is used for a parametric analysis to assess changes to mass and/or stiffness parameters of the structure. Comparing the modes of two different FEMs, identifying the corresponding mode shapes, and tracking the modes for a series of different models is necessary to assess the effects on dynamic responses and loads. Numerical procedures for comparing two mode shapes have been used for many years, including Modal Assurance Criteria (MAC) and CrossOrthogonality (XOR). These techniques compare two mode shapes and return a value from 0 to 1 (or -1 to +1 for

\footnotetext{
${ }^{1}$ Dynamics \& Loads Tech Fellow, Jacobs Engineering, 1500 Perimeter Parkway, Huntsville, AL, AIAA Member.

${ }^{2}$ Structural Dynamics Analyst, Jacobs Engineering, 1500 Perimeter Parkway, Huntsville, AL, AIAA Member.
} 
Cross-Orthogonality) that quantifies the correlation between the two mode shapes. Additional numerical mode shape correlation calculations, based on strain and kinetic energy distributions within the structure, were used as additional comparators. These energy distribution calculations were used to quantify the modal correlation between complex FEMs that might otherwise be difficult to differentiate using traditional XOR or MAC calculations. When incorporated into existing model reduction processes, the energy distribution method provides additional insight into the dynamic characteristics of components that might have been reduced out of the structure, allowing for the correlation of local modes that may not have been captured using the XOR or MAC alone.

For relatively simple structures, the process of identifying corresponding mode shapes between different analysis models can be trivial. However, for complex assemblies such as an integrated launch vehicle with 100's or even 1000 's of modes in the frequency range of interest, this modal tracking effort can be extensive. Adding to this effort is the need to identify corresponding mode shapes across an extremely large set of models. A mode tracking algorithm has been developed to automate this task, which provides a significant time-savings to the structural dynamics engineer.

\section{Numerical Approach}

Finite element based programs such as NASTRAN are used to perform a variety of structural dynamics and loads analyses on a structure. The structural dynamic characteristics of the model (i.e., natural frequencies and mode shapes) are needed to calculate responses and loads from applied forcing functions. An important task in the loads analysis process involves comparing and/or tracking modes for different models. An accepted technique for comparing a pair of mode shapes includes the MAC [1]:

$$
\{M A C\}=\frac{\left[\left\{\mathrm{PHI}_{1}\right\}^{T} \times\left\{\mathrm{PHI}_{2}\right]^{2}\right.}{\left[\left\{\mathrm{PHI}_{1}\right\}^{T} \times\left\{\mathrm{PHI}_{1}\right\}\right] \times\left[\left\{\mathrm{PHI}_{2}\right\}^{T} \times\left\{\mathrm{PHI}_{2}\right\}\right]}
$$

Where: $\quad\left\{\mathrm{PHI}_{1}\right\}=$ Mode Shape Number 1

$$
\left\{\mathrm{PHI}_{2}\right\}=\text { Mode Shape Number } 2
$$

The MAC compares two vectors by calculating the dot or scalar product and dividing by the magnitude of the vectors. This calculation gives the cosine of the angle between the two vectors, yielding a numerical indicator of 1.0 when the angle is 0 or 180 -degrees, and 0.0 when the vectors are orthogonal.

Another accepted numerical technique for comparing a pair of mode shapes includes the Cross-Orthogonality:

$$
\begin{aligned}
& \{\mathrm{XOR}\}=\left\{\mathrm{PHI}_{1}\right\}^{T} \times\{\mathrm{MAA}\} \times\left\{\mathrm{PHI}_{2}\right\} \\
& \text { Where: }\left\{\mathrm{PHI}_{1}\right\}=\text { Mode Shape Number } 1 \\
& \left\{\mathrm{PHI}_{2}\right\}=\text { Mode Shape Number } 2 \\
& \{\text { MAA }\}=\text { Reduced Analytical Mass Matrix }
\end{aligned}
$$

The XOR value between two mode shape vectors can vary from -1.0 to +1.0 , with values of -1.0 or +1.0 showing that the mode shapes are perfectly correlated, and a value of 0.0 indicating that the vectors are uncorrelated.

Both the MAC and XOR are used to help identify corresponding mode shapes between varying FEMs. However, these numerical indicators are sometimes inadequate for comparing analysis mode shapes, especially when model reduction is used. When using the XOR calculation, adequate reduction of the mass matrix is essential to obtain meaningful results, especially when localized or structurally insignificant modes are not retained in the reduced model. The MAC calculation comes with its own limitations that can result in ambiguous numerical indicators that fail to differentiate system modes from localized modes. These limitations may make the XOR and MAC inadequate in identifying the correct correlated mode shapes, requiring additional effort to determine the correct correlated mode shapes (including manually comparing mode shape static or animated plots).

The MAC is a simple technique for comparing two vectors, and is not limited to comparing mode shape vectors. It is possible in NASTRAN to calculate the modal Grid Point Kinetic Energy (GPKE) and Element Strain Energy (ESE). The modal GPKE distribution identifies the significant mode shape mass participation locations, while the modal ESE distribution identifies the significant flexing/elastic strain locations in a mode shape. NASTRAN models can be divided into groups of nodes and elements that make up a structural assembly, based on a logical 
breakdown of the component parts. Using this numerical correlation technique requires that the modal GPKE and/or ESE are calculated for each defined group of nodes or elements and that the energy distributions for these groups are put into vector form. The energy distribution correlations for these vectors are calculated using the same MAC technique defined in Equation [1]. Similar to the mode shape MAC calculation, these energy distribution correlations yield a numerical indicator between 0 and 1, with 1 indicating perfect correlation between the mode shapes and 0 indicating uncorrelated mode shapes. Patran includes the ProCor module [2], which allows the user to easily generate modal strain and kinetic energy distributions (percentage of the total energy for each mode). These energy distributions (either generated from ProCor or manually out-of-code), are then used by the mode tracking tool to calculate ESE and GPKE MAC matrices. These modal energy distribution indicators provide a means, in addition to the MAC and XOR, to compare a set of analytical mode shapes, which is useful in differentiating modes in which the MAC or XOR alone are not adequate. A simple example is presented to show how modal energy distributions differ from modal displacement comparisons for comparing/differentiating two mode shapes. Mode shapes, strain and kinetic energy distributions were calculated for a cantilevered aluminum beam, as shown in Figures 1 and 2.

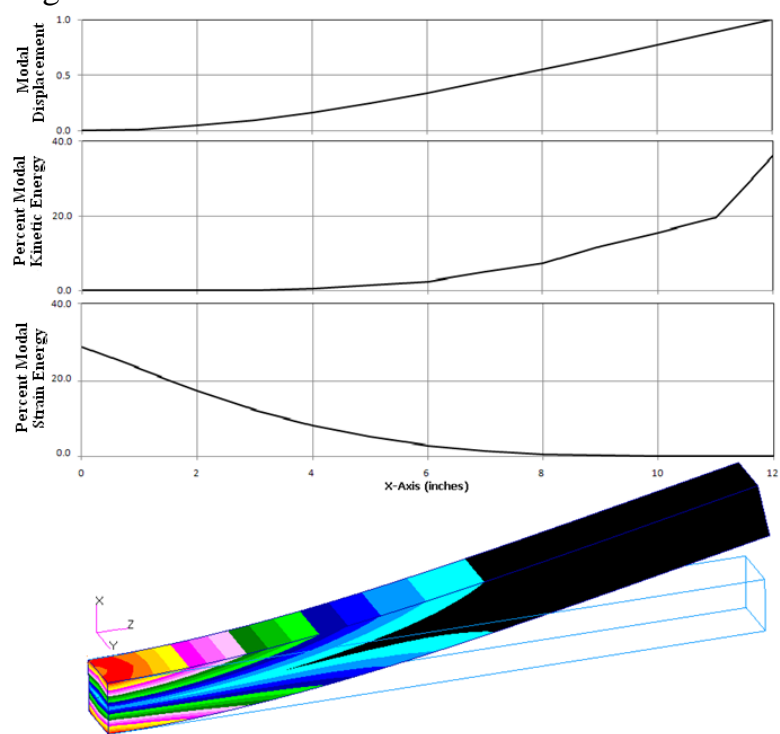

Figure 1. $1^{\text {st }}$ Bending Mode at $218 \mathrm{~Hz}$.

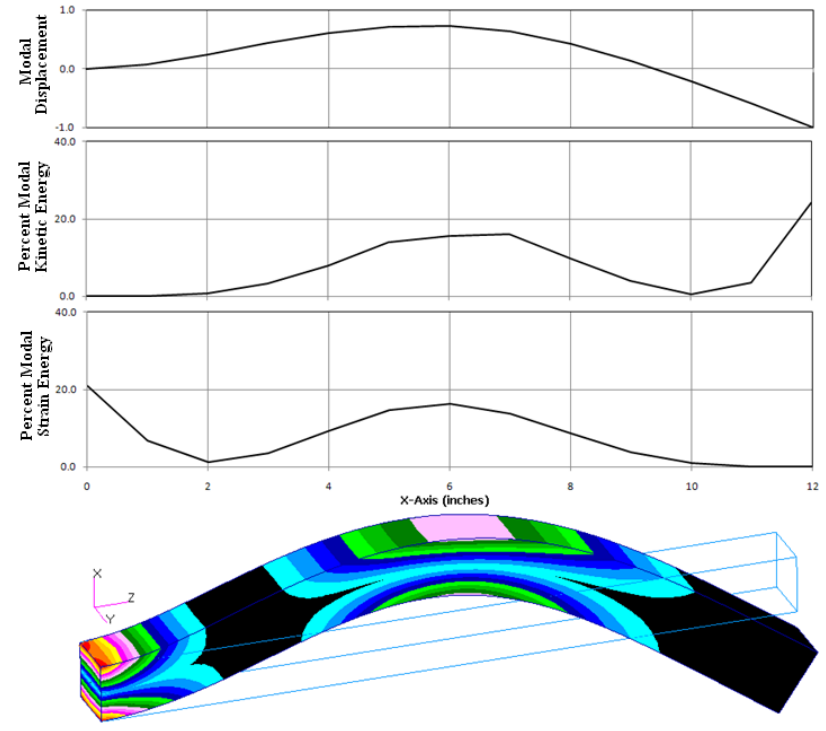

Figure 2. 2nd Bending Mode at $1325 \mathrm{~Hz}$.

These figures demonstrate a difference between the modal kinetic energy distribution (which has a similar character as the modal displacement) and the modal strain energy distribution. For the first bending mode, the maximum modal displacement and kinetic energy occurs at the free end, while the maximum strain energy occurs at the constrained end, which by definition has no modal displacement and/or kinetic energy. This example clearly demonstrates that each of the two modal energy metrics emphasize different regions of a given model. The differences between the strain and kinetic energy distributions provide an added means to differentiate between mode shapes than do the MAC or XOR alone.

\section{Mode Shape Comparison for FEMs with Different Meshes}

It is often necessary to compare and track the modes for FEMs with varying levels of fidelity or different modeling approaches (e.g., using beam elements versus shell elements versus solid elements). It is common to perform an initial loads analysis using a very coarse/simplified model. As the design progresses, and FEM fidelity increases, it is necessary to compare fundamental modes to assess potential loads impacts. When comparing models using traditional MAC and/or XOR techniques, it is necessary to compare mode shapes at the nodes of the coarsest mesh density. Since there is not a one-to-one correspondence of nodes between two models with different mesh densities, the analyst must first select corresponding node pairs. Thus, when comparing a lower-fidelity to a highfidelity FEM, it is necessary to use a smaller subset of the higher-fidelity model nodes for the comparison. The energy distribution MAC comparisons allow the analyst to define groups of nodes and elements that represent specific structural components. To further emphasize this point, consider the design analysis process for a simple electronics box. A coarse FEM used in a preliminary design analysis might represent the electronics box as a single lumped mass, while a higher-fidelity FEM may include a high mesh density to represent local interface fasteners or internal electronic components. The energy distribution MAC approach can easily accomodate these differences in 
mesh density by allowing the analyst to assemble similar nodes and elements into corresponding groups from which the total modal energies can then be calculated and directly compared.

The Marshall Space Flight Center (MSFC) modal test laboratory uses a simple welded aluminum T-plate as a training and demonstration tool. This T-plate has been modal survey tested numerous times, and the resulting frequencies, mode shapes, and modal damping factors are repeatable and readily available. Figure 3 shows that three separate NASTRAN FEMs have been developed to represent this structure, including a beam element mesh, a shell element mesh, and a solid element mesh, each with a significantly different number of grid points in the FEMs.

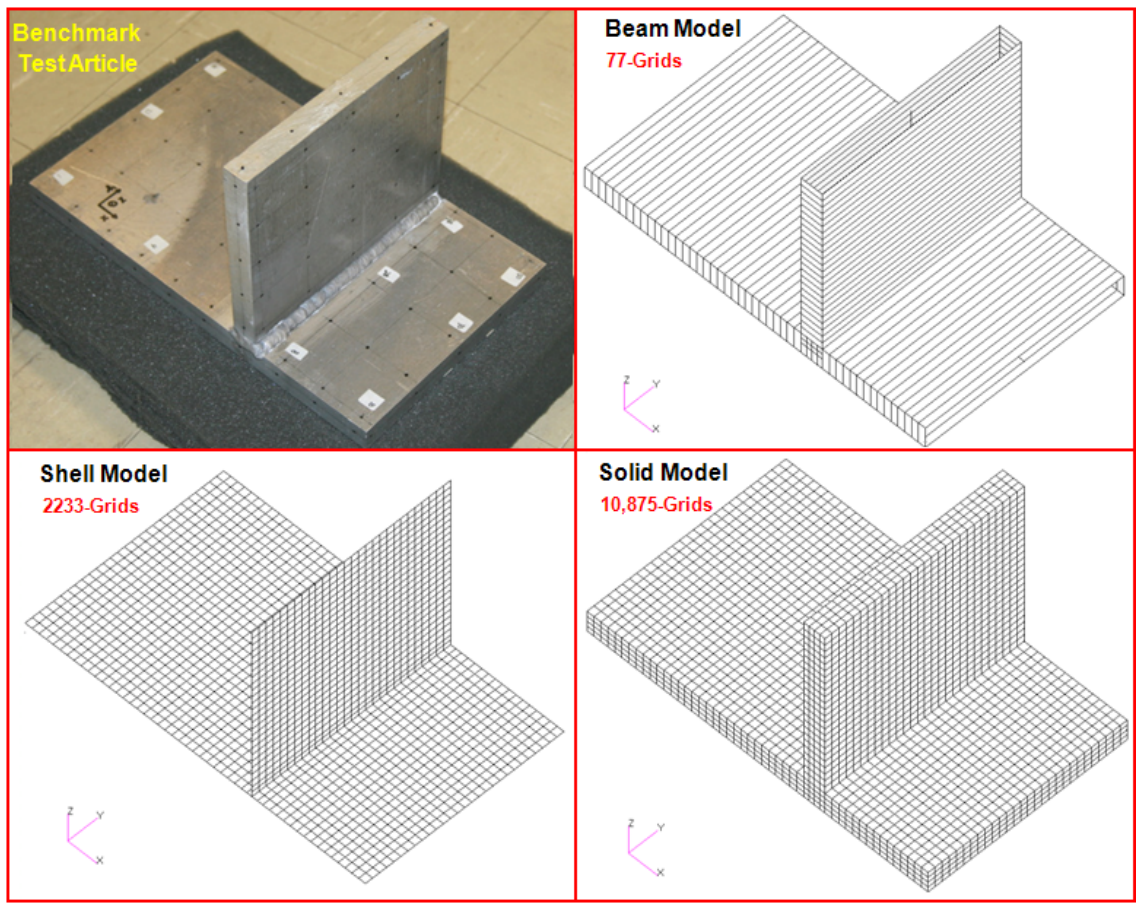

Figure 3. T-Plate with Three Different Element Meshes.

Natural frequencies, mode shapes, modal strain and modal kinetic energy distributions were calculated for the three models with free-free boundary conditions, and the analytical mode shapes were compared using the different numerical techniques described in this paper. Mode shape numerical correlation indicators were used to identify corresponding modes between the different models. Side-by-side mode shape plots including strain energy contours are shown in Figures 4, 5, and 6 for the first three elastic modes. These comparisons demonstrate that the techniques used to compare mode shapes for FEMs with different mesh densities are effective numerical indicators capable of identifying corresponding mode shapes from different FEMs. Mode shapes were compared using the MAC, the GPKE MAC, and the ESE MAC. A single modal correlation metric is calculated between each set of mode shapes, by combining the MAC, the GPKE MAC, and the ESE MAC values using either a Root Sum Squared (RSS) or an algebraic average of the three individual correlation values. The resulting combined metric is a value between 0.0

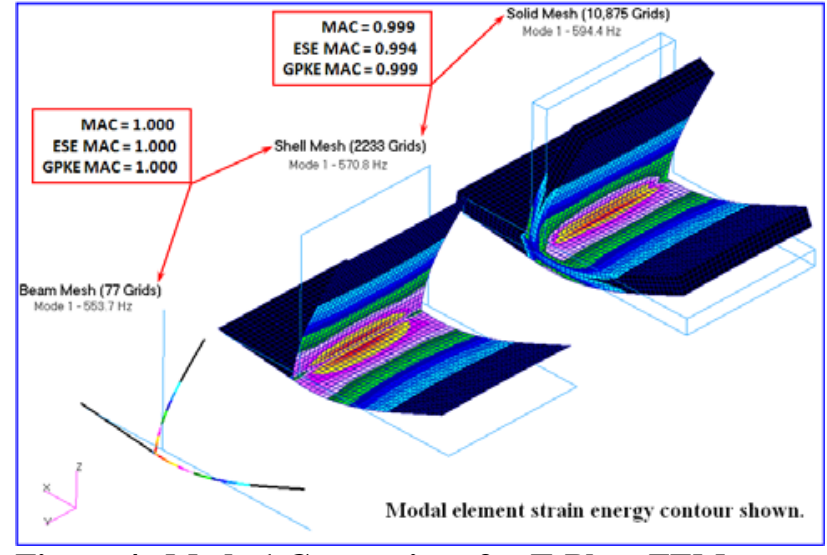

Figure 4. Mode 1 Comparison for T-Plate FEMs.

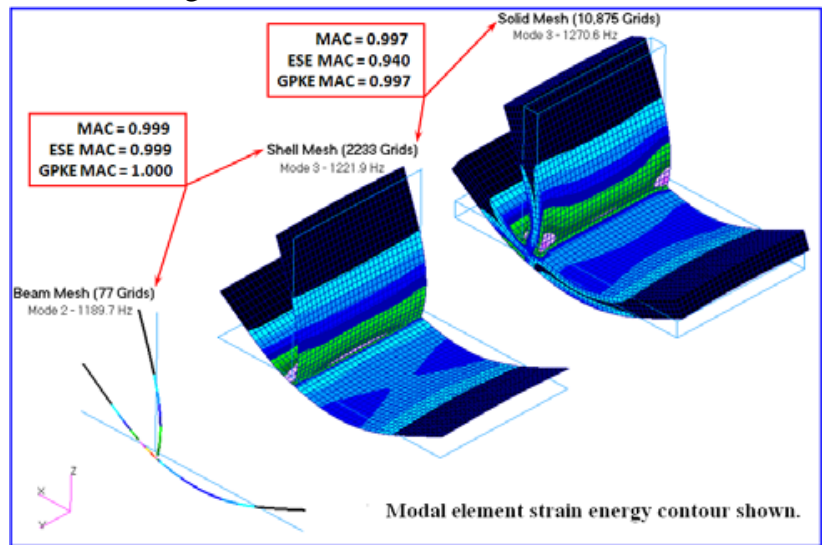

Figure 5. Mode 2 Comparison for T-Plate FEMs.

4

American Institute of Aeronautics and Astronautics 


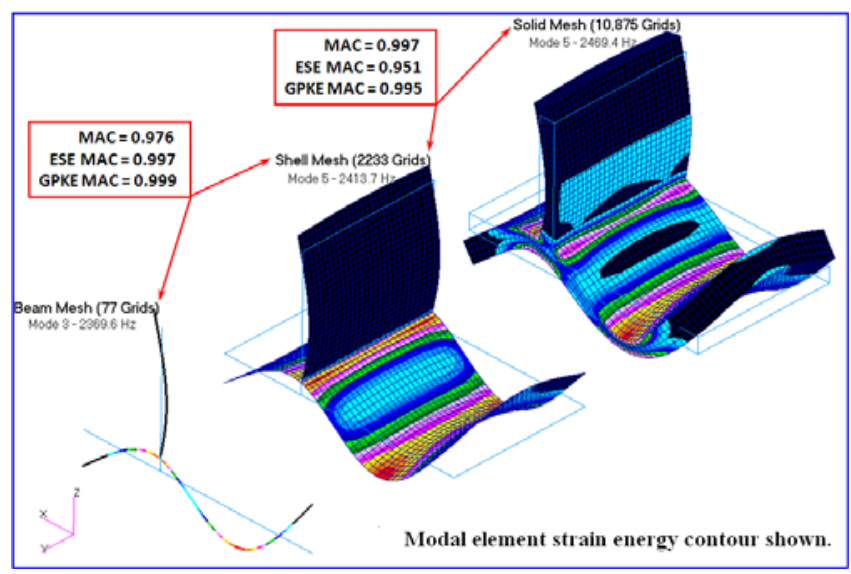

Figure 6. Mode 3 Comparison for T-Plate FEMs. and 1.0, and provides a single measure of the correlation between mode shapes, accounting for modal displacement, kinetic energy, and strain energy distributions.

\section{Automated Mode Shape Tracking}

The process described in this paper provides an improved analysis approach for comparing mode shapes from different FEMs using a combined modal correlation metric calculated from both traditional and energy distribution MACs. This combined metric allows the analyst to accurately identify corresponding mode shapes between models with greater confidence than would have been possible when using a single numerical indicator from the MAC or XOR alone, particularly when working with large, complex and/or reduced FEMs. The additional insight that this improved approach provides with respect to localized modes of a reduced structure also helps to reduce the ambiguity that is sometimes encountered when attempting to track modes from model-to-model. Therefore, this improved analysis approach lends itself well to automation and has been incorporated into a mode tracking algorithm that helps the analyst to quickly establish comprehensive mode mappings for models and/or model groups that would otherwise prove too time consuming and computationally costly to establish manually.

This mode-tracking algorithm has been used to develop a Matlab-based analysis tool that was subsequently used to quickly and accurately track modes for large, complex system models including a full turbopump rocket engine and for an integrated launch vehicle dynamics model with a mass that changes with time during ascent. In addition, this analysis tool was used to track modes for extremely large model sets, allowing for the calculation of statistics even when mode numbers change from model-to-model. The tool is also well suited to automatically track modes

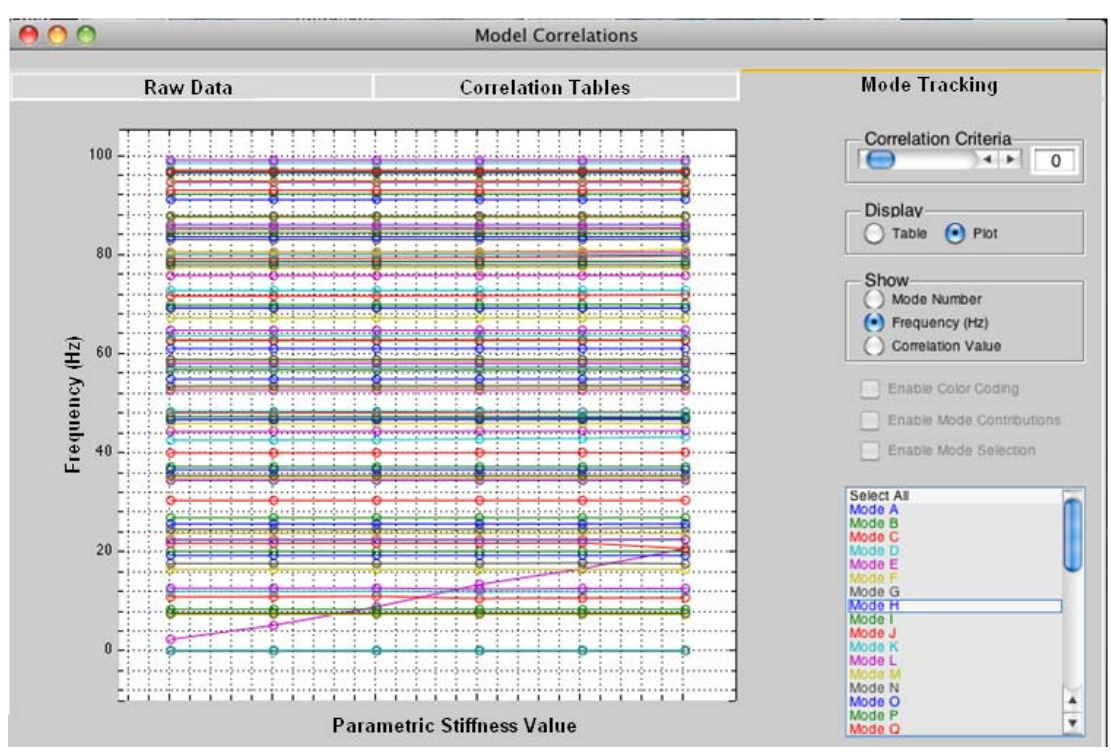

Figure 7. Automated Mode Tracking Results from Parametric Analysis. for a variety of other parametric analyses (i.e., the stiffness of a specific structural component was varied through a range of values), and dispersion analyses (i.e., the mass and stiffness of individual components were varied from the nominal values). A sample mode mapping analysis was performed to demonstrate this automated analysis tool for a parametric stiffness analysis, and the results are shown in Figure 7. 


\section{Conclusion}

A technique was developed and incorporated into an automated analysis tool that compares analysis mode shapes for different FEMs (different mesh densities/element types, design modifications, stiffness parametrics, or different times during a launch vehicle flight), and automatically tracks natural frequencies/mode shapes for these models. Mode shapes for the different analysis models are compared using standard mode shape correlation numerical indicators (MAC and Cross-Orthogonality) as well as by comparing the strain and kinetic energy distributions in the modes using the ESE MAC and the GPKE MAC. A single combined metric is calculated by combining the MAC/XOR, ESE MAC, and GPKE MAC, and the modes with the highest correlation value are identified as corresponding mode shapes in the different models. The addition of modal strain and kinetic energy distribution comparisons in the mode shape comparisons provides a more reliable method of identifying and tracking corresponding modes in different analytical models, especially for systems with a large modal density and significant modal coupling. The automated analysis tool has been used to track modes for several large launch vehicle and propulsion system NASTRAN models for parametric stiffness analyses and mass and/or stiffness dispersion analyses.

\section{Acknowledgments}

Robert Towner and Jonathan Band thank Jack Stockdale/Jacobs ESTS Group Director of Engineering for supporting this analysis tool development effort. They also thank Scott Joji/Qualis Corporation and the MSFC ET40 Modal Test Lab for providing test results for the T-Plate used to demonstrate the automated mode tracking analysis tool.

\section{References}

${ }^{1}$ Ewins, D. J., Modal Testing Theory, Practice, and Application, $2^{\text {nd }}$ ed., Research Studies Press, Ltd., 2000, pp. $422-424$.

${ }^{2}$ MSC.Software Corporation, MSC.ProCOR 2006 User’s Guide, 2006. 